\title{
SOME APPLICATIONS OF A THEOREM OF RADO
}

\section{J. A. WELSH}

It has been shown by Mirsky and Perfect [1] that the theorem of Rado [2], linking matroid theory and transversal theory, has important applications in combinatorial theory. In this note I use it to obtain necessary and sufficient conditions for two families of sets to have a common transversal containing a given set, and then I show how it may be used to obtain a variant of a well-known theorem that was obtained by Hoffman and Kuhn [3] using linear programming methods.

I use the notation of Mirsky and Perfect [4]. If $\mathbf{A}=\left\{A_{i} ; i \in I\right\}$ is any finite family of sets, the set of distinct elements $\left\{x_{i} ; i \in I\right\}$ is a transversal of $\mathbf{A}$ if $x_{i} \in A_{\sigma(i)}$, ( $i \in I$ ), for some permutation $\sigma$ of $I$. If $\mathbf{A}=\left\{A_{i} ; i \in I\right\}$ and $\mathbf{B}=\left\{B_{i} ; i \in I\right\}$ are two finite families with the same index set, the set of distinct elements $\left\{x_{i} ; i \in I\right\}$ is a common transversal of $\mathbf{A}$ and $\mathbf{B}$ if it is both a transversal of $\mathbf{A}$ and a transversal of $\mathbf{B}$. (Although the individual sets $A_{i}, B_{i}$ are not assumed to be finite, in practice this assumption may usually be made without loss of generality. This is because the various conditions considered will clearly hold for infinite sets if and only if they hold for suitably chosen finite subsets.)

Hoffman and Kuhn [5], completing a result of Mann and Ryser [6], gave necessary and sufficient conditions for $\mathbf{A}=\left\{A_{i} ; i \in I\right\}$ to have a transversal which contains a given finite set $C$, namely that for all subsets $J$ of $I$

$$
\min (A(J),|I|-|C-A(J)|) \geqslant|J|,
$$

where we use $A(J)$ to denote the union of those $A_{i}$ whose indices are members of $J$. These conditions are clearly necessary; it would be easy to prove their sufficiency by applying Rado's theorem to the matroid $(A(I), \mathbf{M})$ where $X \in \mathbf{M}$ if $|X \cup C| \leqslant|I|$. Mirsky and Perfect [1] have given a proof based on the notion of deltoid independence.

Ford and Fulkerson [7] gave necessary and sufficient conditions for $A=\left\{A_{i} ; i \in I\right\}$ and $B=\left\{B_{i} ; i \in I\right\}$ to have a common transversal, namely that

$$
|A(J) \cap B(K)| \geqslant|J|+|K|-|I|
$$

for all subsets $J, K$, of $l$. Mirsky and Perfect [1] give a proof based on Rado's theorem.

I first prove a common generalisation of the above two results.

THeorem 1. Two families $\mathbf{A}$ and $\mathbf{B}$, with common finite index set $I$, have $a$ common transversal which contains the set $C$, if, and only if,

$$
|\{A(J) \cap B(K)\}-C|+|A(J) \cap C|+|B(K) \cap C| \geqslant|J|+|K|+|C|-|I|
$$

for all subsets $J, K$ of $I$.

We recover the result of Ford and Fulkerson by taking $C=\varnothing$ and that of Hoffman and Kuhn [5] on taking $B_{k}=A(I)$ for all $k \in I$ and considering the two cases $K=\varnothing, K \neq \varnothing$. 
Before proving Theorem 1, I review the matroid terminology used. A matroid $(S, \mathbf{M})$ is a family $\mathbf{M}$ of independent subsets of a finite set $S$ such that:

(1) $\varnothing \in \mathbf{M}$;

(2) $X \in \mathbf{M}$ and $Y \subset X$ implies $Y \in \mathbf{M}$;

(3) if $Z$ is any subset of $S$, the maximal independent subsets of $Z$ have the same cardinality. This cardinality is called the rank of $Z$ in $(S, \mathbf{M})$ and will be denoted by $r(Z)$.

A base of $(S, M)$ is a maximal independent subset of $S$, and alternative axioms defining a matroid in terms of its bases are given by Whitney [8]:

(4) no proper subset of a base is a base;

(5) if $B, B^{\prime}$, are bases and $e \in B$, then there exists $f \in B^{\prime}$ such that $B-e+f$ is a base.

For further details of matroid theory see [8] or Tutte [9]. We state now the result of Rado [2] which is the central theorem on which this paper is based.

RAdo's TheOREM. If $(S, \mathbf{M})$ is a matroid, and $\mathbf{A}=\left\{A_{i} ; i \in I\right\}$ is any family of subsets of $S$, then $\mathbf{A}$ has a transversal which is independent in the matroid $\mathbf{M}$, if, and only if, for all $J \subset I$

$$
r(A(J)) \geqslant|J|
$$

Proof of Theorem 1. We may suppose that $A(I)$ is finite. Let $\mathbf{T}$ denote the set of transversals of the family A. Then by a theorem of Edmonds and Fulkerson [10] (also obtained independently by Mirsky and Perfect [1]), $\mathbf{T}$ is the set of bases of a matroid on $S$, which we call the transversal matroid determined by $\mathbf{A}$ and denote by $[S, \mathbf{T}(\mathbf{A})]$. (We allow $\mathbf{T}$ to be the null set in which case $[S, \mathbf{T}(\mathbf{A})]$ is the trivial matroid having the null set as its only base.)

Define a new matroid $(S, \mathbf{T})$ on $S$ by the rule that a subset $X$ is a base of $\mathbf{T}$ if and only if $X$ is a base of $[S, T(A)]$ and in addition $X$ contains the set $C$. It is trivial to show that this does in fact define a matroid on $S$ by axioms (4) and (5). It is clear that $\mathbf{A}$ and $\mathbf{B}$ have a common transversal containing the set $C$ if and only if $\mathbf{B}$ has $\dot{\mathbf{a}}$ transversal which is a base of the matroid $(S, T)$. Hence by the theorem of Rado, A, B have a common transversal containing $C$ if, and only if,

(6) $r(B(K)) \geqslant|K|$

where $r()$ is the rank function of the matroid $(S, \mathbf{T})$, and $K$ is any subset of $I$. Since any independent set in a matroid can be augmented to a base, it is not difficult to see that for any subset $X$ of $S$

$$
r(X) \geqslant k \text {, }
$$

if and only if $X \cup C$ contains a partial transversal of $\mathbf{B}$ of cardinality not less than

$$
k+|C|-|C \cap X| \text {. }
$$

This means that

$$
\left\{(X \cup C) \cap B_{i} ; \quad i \in I\right\}
$$

must have a partial transversal with defect

$$
|I|-k-|C|+|C \cap X| \text {. }
$$


By a theorem of Ore [11] the condition for this is that

$$
|(X \cup C) \cap B(K)| \geqslant|K|-|I|+k+|C|-|C \cap X|
$$

for any subset $K$ of $I$.

Hence (6) is satisfied if, and only if,

$$
|(A(J) \cup C) \cap B(K)| \geqslant|J|+|K|+|C|-|I|-|C \cap A(J)|
$$

for any subsets $J, K$, of $I$.

It is trivial to put (7) into the symmetric form used in the statement of the theorem.

Another application of Rado's theorem is to prove

THEOREM 2. If $\left\{E_{i} ; i \in J\right\}$ is any partition of a finite set $S$ into disjoint subsets, and $\mathbf{A}=\left\{A_{i} ; i \in I\right\}$ is any finite family of subsets of $S$, then $A$ has a transversal $X$ such that

$$
\left|X \cap E_{j}\right| \leqslant a_{j}, \quad j \in J,
$$

where the $a_{j}, j \in J$, are prescribed integers, if, and only if,

$$
|A(K)|-\sum_{J}\left(\left|A(K) \cap E_{j}\right|-a_{j}\right)^{+} \geqslant|K|
$$

for all subsets $K$ of $I$ (where, as in Mirsky and Perfect [4], we write $z^{+}$for $\max (z, 0)$ ).

This theorem, although only a weaker form of a theorem of Hoffman and Kuhn [3], seems worth mentioning because of the very simple proof.

Proof. Let $(S, M)$ be the matroid which has as bases those subsets $Z$ of $S$ which satisfy

$$
\left|Z \cap E_{j}\right|=a_{j}, \quad(j \in J) .
$$

It is trivial, using axioms (4) and (5), to verify that this is in fact a matroid. Now if $r($.$) denotes the rank function in (S, M)$ then for any $X \subset S$

$$
r(X)=|X|-\sum_{J}\left(\left|X \cap E_{j}\right|-a_{j}\right)^{+} .
$$

But the family $\mathbf{A}$ has a transversal $X$ which satisfies

$$
\left|X \cap E_{j}\right| \leqslant a_{j}, \quad(j \in J),
$$

if, and only if, $\mathbf{A}$ has a transversal $X$ which is an independent set in $(S, \mathbf{M})$. By Rado's theorem this is so if and only if

$$
r(A(K)) \geqslant|K|
$$

for all $K \subset I$. The theorem follows from (8).

Using the same technique we now establish an alternative version of the main theorem of Hoffman and Kuhn [3].

Let $\mathbf{E}=\left\{E_{j} ; j \in J\right\}$ be any partition of the finite set $S$ into disjoint subsets. Let $a_{j}, b_{j},(j \in J)$ be integers with

$$
0 \leqslant a_{j} \leqslant b_{j}, \quad(j \in J) .
$$

For any subset $X$ of $S$ we define a lower deficiency $L(X)$ by

$$
L(X)=\sum_{j}\left(a_{j}-\left|X \cap E_{j}\right|\right)^{+}
$$

and an upper deficiency $H(X)$ by

$$
H(X)=\sum_{J}\left(\left|X \cap E_{j}\right|-b_{j}\right)^{+} .
$$




\section{We prove}

THEOREM 3. Let $A=\left\{A_{i} ; i \in I\right\}$ be a finite family of subsets of a finite set $S$. Let $\left\{E_{j} ; j \in J\right\}$ be any partition of $S$ into pairwise disjoint subsets. Let $a_{j}, b_{j},(j \in J)$ be integers such that

$$
0 \leqslant a_{j} \leqslant b_{j}, \quad(j \in J) .
$$

Then $\mathbf{A}$ has a transversal $X$ with the property

$$
a_{j} \leqslant\left|X \cap E_{j}\right| \leqslant b_{j}, \quad(j \in J),
$$

if and only if for all $K \subset I$

$$
(|I|-|A(K)|-L(A(K))+H(A(K)))^{+} \leqslant|I|-|K|-L(A(K)) .
$$

The conditions given are at first sight unattractive but from a computational point of view they are certainly not more formidable than those given by Hoffman and Kuhn.

Proof. Let $(S, M)$ be the matroid having as bases only those subsets $B$ of $S$ such that

$$
\begin{gathered}
|B|=|I|, \\
a_{j} \leqslant\left|B \cap E_{j}\right| \leqslant b_{j} .
\end{gathered}
$$

We show that the family $\mathbf{B}$ of subsets of $S$ satisfying (10) and (11) also satisfy the base axioms of a matroid. Clearly no member of $\mathbf{B}$ can properly contain another. Let $B_{1}, B_{2}$ be distinct members of $\mathbf{B}$ and let $e \in B_{1}$. Since $\left\{E_{j} ; j \in J\right\}$ partition $S$, $e$ is a member of exactly one $E_{j}$, say $E_{1}$. Hence

$$
a_{1}-1 \leqslant\left\{\left(B_{1}-e\right) \cap E_{1} \mid \leqslant b_{1}-1 .\right.
$$

If $B_{2} \cap E \notin\left(B_{1}-e\right) \cap E_{1}$, let $f \in\left(B_{2} \cap E_{1}\right)-\left(B_{1} \cap E_{1}\right)$, and $B_{3}=B_{2}-e+f$ satisfies (10) and (11).

If $\left(B_{2} \cap E\right) \subset\left(B_{1}-e\right) \cap E_{1}$, then since $B_{2}$ satisfies (11),

$$
a_{1} \leqslant\left|\left(B_{1}-e\right) \cap E_{1}\right| \leqslant b_{1}-1 \text {. }
$$

Also, since $\left|B_{2}\right|=\left|B_{1}-e\right|+1$, there must exist some $E_{j}(j \neq 1)$ such that

$$
\left|\left(B_{1}-e\right) \cap E_{j}\right|<\left|B_{2} \cap E_{j}\right| \leqslant b_{1} .
$$

Let $f \in\left(B_{2} \cap E_{j}\right)-\left(B_{1}-e\right)$; then clearly $B_{1}-e+f$ satisfies (10) and (11) and thus $(S, M)$ is a matroid.

By the definition of $(S, \mathbf{M})$, the family $\mathbf{A}$ has a transversal which satisfies the intersection properties (9) if and only if $\mathbf{A}$ has a transversal which is a base of $(S, \mathbf{M})$. Again using Rado's theorem this will be so if, and only if, for any $K \subset I$,

where $r()$ is the rank function of $(S, \mathbf{M})$.

$$
r(A(K)) \geqslant|K|
$$

Now if $X$ is any subset of $S, r(X) \geqslant u$ if, and only if, there exists a subset $Y$ of $S$ such that $X \cup Y$ contains a base of $(S, \mathbf{M})$, and

$$
|Y| \leqslant r(S)-u=|I|-u \text {. }
$$

But this subset $Y$ must contain

elements.

$$
L(X)+(|I|-|X|-L(X)+H(X))^{+}
$$


Hence $r(X) \geqslant u$ if and only if

$$
L(X)+(|I|-|X|-L(X)+H(X))^{+} \leqslant|I|-u .
$$

This proves the theorem.

Conclusion. The purpose of this paper is to illustrate the power of the theorem of Rado, proved as early as 1942. Many other theorems on transversal theory proved by methods of linear programming or ad hoc combinatorial methods may be deduced from it, and it certainly appears to have a strong claim to being one of the fundamental theorems of combinatorial theory.

Acknowledgment. Since proving the above theorems I have been shown by Miss Hazel Perfect an alternative proof of Theorem 1 based on Menger's graph theorem [12], and Dr. L. Mirsky has pointed out to me that it is not difficult to transform the conditions I give in Theorems 2 and 3 to those given by Hoffman and Kuhn in their original paper. I am grateful for these and other communications from Miss Perfect and Dr. Mirsky. I should also like to acknowledge some very helpful comments by the referee.

\section{References}

1. L. Mirsky and Hazel Perfect, "Applications of the notion of independence to problems in combinatorial analysis ", Journ. Comb. Theory, 2 (1967), 327-357.

2. R. Rado, "A theorem on independence relations", Quart. J. Math. (Oxford), 13 (1942), 83-89.

3. A. J. Hoffman and H. W. Kuhn, "On systems of distinct representatives; Linear inequalities and related systems", Ann, Math. Studies, 38 (Princeton, 1956), 199-206.

4. L. Mirsky and Hazel Perfect, "Systems of representatives", J. Math. Anal. and Appl., 15 (1966), 520-568.

5. A. J. Hoffman and H. W. Kuhn, "Systems of distinct representatives and linear programming ", Amer. Math. Monthly, 63 (1956), 455-460.

6. H. B. Mann and H. J. Ryser, "Systems of distinct representatives ", Amer."Math. Monthly, 60 (1953), 397-401.

7. L. R. Ford, Jr. and D. R. Fulkerson, Flows in Networks (Van Nostrand, Princeton, 1962).

8. H. Whitney, "On the abstract properties of linear dependence ", Amer. J. Math.. 57 (1935), 509-533.

9. W. T. Tutte, "Lectures on matroids", Journ. N.B.S., 69B (1965), 1-48.

10. J. Edmonds and D. R. Fulkerson, "Transversals and matroid partition ", Journ. N.B.S., 69B (1965), 147-153.

11. O. Ore, "Graphs and matching theorems ", Duke Math. Journ., 22 (1955), 625-639.

12. Hazel Perfect, "Some applications of Menger's graph theorem ", J. Math. Anal. and Appl., (to appear).

Merton College, Oxford.

and

The University of Michigan,

(Received on the 20th of February, 1968.) 